\title{
PLANO DE SAÚDE CORPORATIVO:
}

estudo de validação de um instrumento de avaliação da qualidade em uma indústria de calçados

\section{1- Fernando Dewes*}

Doutor em Psicologia pela Pontifícia Universidade Católica do Rio Grande do Sul (PUC/RS).

Professor da Universidade de Caxias do Sul (UCS)

fdewes@ucs.br

http://lattes.cnpq.br/8651751640164983 


\title{
PLANO DE SAÚDE CORPORATIVO: ESTUDO DE VALIDAÇÃO DE UM INSTRUMENTO DE AVALIAÇÃO DA QUALIDADE EM UMA INDÚSTRIA DE CALÇADOS
}

\section{RESUMO}

Comumente a avaliação da qualidade de um plano de saúde corporativo considera apenas o grau de satisfação dos seus usuários. Para a avaliação, tanto do grau de satisfação do usuário quanto do retorno do investimento em planos de saúde para a empresa, procedeu-se a construção e a validação de uma escala multidimensional. A construção da escala levou em conta as dimensões da qualidade associadas à satisfação do usuário e ao impacto dessa satisfação na sua relação com a empresa, como uma qualidade agregada. Para validação da escala foi procedida uma análise das suas propriedades psicométricas, a partir dos dados gerados por uma amostra de 280 trabalhadores. Os resultados mostram que o plano de saúde proporciona satisfação aos trabalhadores e ganhos para a empresa, de acordo com o Modelo de Qualidade Agregada proposto. A escala apresentou consistência interna total e validade convergente aceitáveis. A análise fatorial sugeriu um conjunto de dez fatores relacionados à qualidade técnica, funcional e agregada do plano de saúde, que respondem por cerca de $60 \%$ da variância total. O estudo reafirma a importância de avaliar o retorno para a empresa dos investimentos em planos de saúde, no que o instrumento desenvolvido neste trabalho poderá ser útil.

\section{Palavras-Chave}

Gestão de pessoas; plano de saúde corporativo; avaliação da qualidade.

\section{CORPORATE HEALTH: A VALIDATION STUDY OF AN INSTRUMENT OF QUALITY ASSESSMENT IN A SHOE INDUSTRY}

\begin{abstract}
Usually, quality assessments of corporative health insurance plans take into account just the degree of satisfaction of the costumers. For the assessment of both the degree of costumer's satisfaction and the firm investment returns, we carried out the construction and a validation test of an appropriate multidimensional measurement scale. For the construction of the scale we took into account the quality dimensions related to the costumer's satisfaction and its impact on the relationship of the employee towards the firm as an aggregated quality asset. As validation test of the proposed scale we conducted an analysis of its psychometric properties based on data generated from a sample of 280 employees. The results show that the health insurance plan bring both satisfactions to the employees and gains to the firm, according to the proposed Model of Aggregated Quality. The proposed measurement scale presented acceptable total internal consistency and convergent validity. The factorial analysis suggested a set of ten factors related to the technical, functional, and aggregated qualities of the health insurance plan, which respond for about $60 \%$ of the total variance. This study stresses the importance of the assessment of the returns to the firm of its investments on health insurance plans, for which the instrument we developed in this paper can be useful.
\end{abstract}

\section{Keywords}

Personnel management; employer-sponsored health plan; quality assessment. 


\section{Introdução}

Os problemas no atendimento através do Sistema Único de Saúde (SUS) e os altos custos dos serviços prestados por clínicas e hospitais particulares são fatores de intranquilidade para a maioria dos brasileiros. Para contornar as dificuldades de um sistema de saúde precário e oneroso foram criadas algumas alternativas, dentre elas a medicina de grupo. Por isto, paralelamente ao SUS, verificou-se um crescimento expressivo dos planos de saúde. Em dezembro de 2003 o número de pessoas conveniadas com algum plano de saúde era de 31.771.197. Em junho de 2010 este número atingiu 44 milhões de beneficiários, tendo as empresas operadoras de planos de saúde uma receita, em 2009, da ordem de 64 bilhões de reais (http://www.ans.gov.br).

No esforço de proporcionar mais tranqüilidade aos seus trabalhadores e ao mesmo tempo amenizar as problemáticas humanas decorrentes de enfermidades, com todas as suas conseqüências sobre a produtividade, muitas empresas passaram também a oferecer planos de saúde aos seus trabalhadores. Para se ter uma idéia da magnitude do fenômeno, o gasto com saúde nas empresas já no ano de 1996, foi estimado em 0,7\% do PIB (Corrêa, Piola \& Arilha, 1998). Estendendo-se este cálculo para 1998, chega-se ao valor de seis bilhões e duzentos e noventa e nove milhões de reais aplicados em planos de saúde pelas empresas brasileiras (Kilsztajn, Câmara \& Carmo, 2001), confirmando a relevância histórica e econômica deste tema. De acordo com a Associação Brasileira de Medicina de Grupo (ABRAMGE, http://www.abramge.com.br) o número de empresas contratantes de planos de saúde em 2009 era da ordem de 52.000. Segundo a AGENCIA NACIONAL DE SAÚDE SUPLEMENTAR (ANS, http://www.ans.gov.br), em junho de 2010 chegou-se ao impressionante número de 24.930 .019 beneficiários de planos coletivos empresariais.

Como consequência da importância do plano de saúde para os trabalhadores e dos altos valores arcados pela empresa para custeá-lo, faz-se necessário avaliar cuidadosamente o retorno deste investimento identificando, não só a qualidade intrínseca dos serviços inerentes ao plano, mas também o seu impacto sobre as atitudes dos trabalhadores. Os dirigentes das empresas necessitam saber se os trabalhadores estão avaliando favoravelmente o benefício recebido e se os reflexos deste benefício se traduzem também em maior motivação para o trabalho, menores índices de absenteísmo e de rotatividade, entre outros indicadores organizacionais. Se assim não o for, ao invés de tornar-se fator de satisfação, o plano de saúde produzirá frustrações com os prováveis reflexos negativos na motivação e produtividade da empresa.

Com o propósito de contribuir para o desenvolvimento de critérios de avaliação da qualidade de serviços de saúde, este artigo apresenta uma escala de medida da qualidade de um plano de saúde proporcionado pela empresa aos seus trabalhadores, bem como as etapas envolvidas na sua construção e validação. Através deste instrumento poderão ser obtidas informações, não só sobre a qualidade dos serviços prestados pelos operadores do plano, mas também sobre o impacto do mesmo nas atitudes dos trabalhadores relacionadas ao seu trabalho e à empresa que Ihes proporciona tal benefício. A ampliação do escopo da mensuração da qualidade de um plano de saúde na empresa é importante para os gestores aferirem o grau de retorno, para a empresa, do investimento envolvido, orientando-os em suas futuras decisões relativas a este benefício.

\section{Modelo de avaliação da qualidade do plano de saúde da empresa}

A avaliação de qualquer serviço, como se sabe, é extremamente complexa, porquanto a sua qualidade é um construto intangível e abstrato, difícil de definir e avaliar (Cronin \& Taylor, 1992). A dificuldade se agrava ainda mais quando o serviço é de natureza médica. A pesquisa mostra que a avaliação da qualidade técnica, por exemplo, pode ser enormemente dificultada porque muitos pacientes não têm o conhecimento necessário para avaliar a qualidade do diagnóstico e da intervenção terapêutica (Bowers, et al, 1994). Conseqüentemente, muitos pacientes baseiam sua avaliação da qualidade técnica mais em aspectos do relacionamento interpessoal e do ambiente onde o serviço é prestado, justamente aqueles sobre os quais os médicos atribuem menos valor (Lam, 1997). Uma boa relação humana e comunicação com o médico poderão, portanto, ser interpretadas como sinais de uma boa qualidade técnica (Spicer, 2002). Problematizando ainda mais a avaliação da qualidade em serviços de saúde, Righi, Schmidt e Venturini (2010) comentam que o processo envolve tanto quem produz os serviços quanto quem os utiliza, porque mesmo que ocupem posições diferentes, ambos contribuem na execução dos serviços, influenciando assim na qualidade dos mesmos. 
A percepção de algo é subjetiva e abrange vários processos psicológicos de uma pessoa, desde as suas sensações até a recuperação de informações armazenadas na sua memória. De acordo com a teoria da percepção construtiva (Sternberg, 2008), durante a percepção de algo construímos e testamos várias hipóteses relativas aos perceptos, baseados no que sentimos (sensações), no que sabemos (conhecimentos prévios armazenados na memória) e nas expectativas com relação àquilo que está sendo considerado. Levando as proposições desta teoria para o campo da avaliação, será possível concluir que a percepção da qualidade de um serviço de saúde resulta do conhecimento do paciente relativamente aos serviços médicos em geral e da comparação de suas expectativas de qualidade com os resultados efetivamente experimentados por ele. Esta posição é sustentada por Gronroos (1995), segundo o qual um serviço de saúde apresenta qualidade percebida quando ele encontra ou excede o nível de expectativa do paciente.

As percepções da qualidade dos serviços de cuidado da saúde podem ser consideradas também em dois focos: qualidade técnica e qualidade funcional (Donabedian, 1980). A qualidade técnica é definida como o grau de precisão e acerto dos diagnósticos e procedimentos médicos aplicados pelos profissionais da saúde. Qualidade funcional, por sua vez, se refere à maneira pela qual os cuidados de saúde são prestados ao paciente, especialmente no aspecto do relacionamento interpessoal, incluindo o tratamento humano, presteza e cortesia.

Outra particularidade da percepção da qualidade de um serviço se refere a possibilidade de ser considerada uma atitude, já que ela envolve cognições, sentimentos e tendências para agir de um determinado modo em relação aos prestadores de um serviço e a tudo o mais que estiver com ele relacionado. A posição segundo a qual a avaliação da qualidade percebida é melhor conceituada como uma atitude, é suportada pela literatura (Cronin \& Taylor, 1992), justificando assim o uso de medidas de atitudes para o julgamento de dimensões específicas da qualidade percebida de um serviço de saúde.

Por outra parte, em se tratando de avaliar a qualidade de um plano de saúde oferecido pela empresa, surge a necessidade de ampliar as dimensões para além da qualidade técnica e funcional. Por esta razão, o modelo sugerido neste estudo passa a ter a seguinte configuração:

Figura 1: Modelo de qualidade agregada de um plano de saúde empresarial

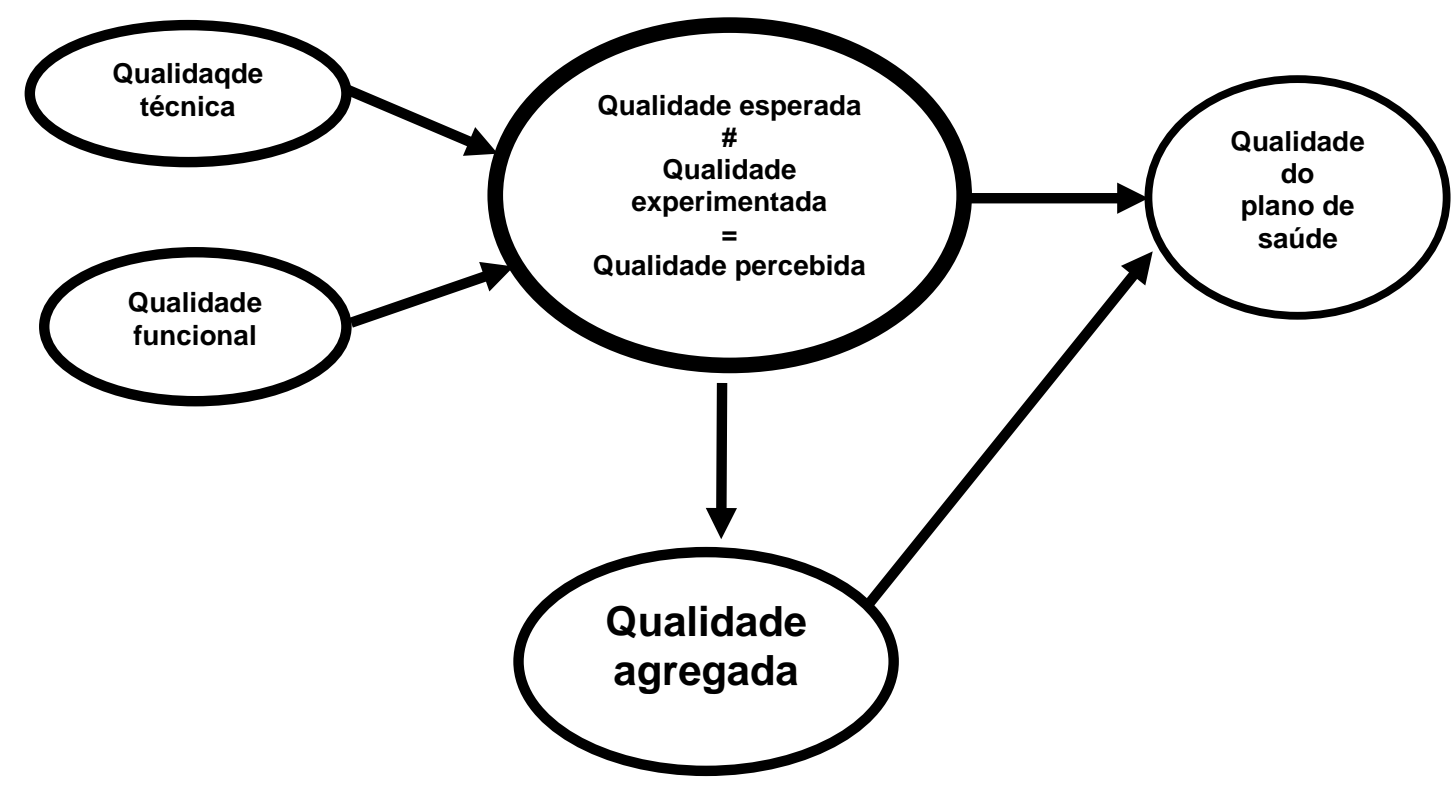

Fonte: Os autores

De acordo com este modelo, a qualidade percebida se expressa pela diferença entre a qualidade esperada e a qualidade experimentada (Gronroos, 1995). A qualidade agregada aparece, então, como efeito da qualidade percebida, constituindo-se na dimensão do plano de saúde que se expressa pelo seu poder de gerar valor adicional para a empresa e para o trabalhador. Estimular maior dedicação ao trabalho, reduzir o absenteísmo e proporcionar uma melhor qualidade da vida, são exemplos de agregação de valor. O pressuposto básico deste modelo é o da existência de uma relação entre qualidade percebida e 
qualidade agregada de tal sorte que, quanto maior a qualidade percebida, maior será a qualidade agregada. Nesta perspectiva, a qualidade do plano de saúde da empresa será, portanto, uma função da qualidade percebida e da qualidade agregada.

\section{Técnicas de avaliação da qualidade de serviços de saúde}

Para avaliar a qualidade de um plano de saúde será preciso consultar as pessoas diretamente envolvidas com a questão. A consulta poderá ser realizada através de consultas individuais, ou em grupos, aplicando-se técnicas específicas de levantamento de opiniões. Gerschman et al (2007) realizaram discussões em grupo de usuários de planos de saúde. As sessões envolviam de sete a dez pessoas que debatiam sobre o tema em questão. Os debates eram moderados por um profissional e cada sessão tinha uma duração média de duas horas. Através da técnica de discussão grupal os autores observaram que foi possível avaliar a satisfação dos beneficiários com os diversos aspectos do serviço de saúde e identificar aqueles aspectos que mais contribuíram para a satisfação dos usuários.

Todavia, como técnica de identificação de percepções relacionadas aos serviços de saúde proporcionados pela empresa, as entrevistas individuais ou técnicas grupais trazem algumas limitações e desvantagens, nem sempre compensadas por seus pontos mais fortes. Elas podem ser demoradas, exigem condições ambientais especiais, não garantem o anonimato do trabalhador entrevistado e seus resultados poderão, por isto, estar distantes dos reais sentimentos ou opiniões das pessoas envolvidas. Outros métodos poderão ser utilizados, como o da caixa de sugestões ou de registro e tratamento de reclamações. Mas estes também apresentam suas limitações. Eles podem ter problemas de representatividade da amostra ou referir-se apenas a alguns aspectos específicos dos serviços a serem avaliados, deixando de considerar outros que são também importantes.

Na busca de técnicas mais efetivas para avaliar a qualidade de serviços de saúde, aparecem às escalas de atitudes como aquelas capazes de fornecerem maior praticidade e confiabilidade. Mesmo sendo de elaboração e validação complexas, seus benefícios poderão compensar eventuais limitações, ao menos quando comparada com outras técnicas de coleta e análise de dados. Sua aplicação é relativamente simples e rápida e os dados por ela fornecidos são apurados e interpretados com facilidade. Embora existam diferentes tipos de escalas de atitudes, o formato da escala de Likert tem mostrado vantagens na redução de erros de percepção sobre outros tipos de escalas. Meric (1994), por exemplo, encontrou menos erros de leniência na avaliação da qualidade de serviços de saúde através de escalas de Likert, na comparação com escalas de formatos mistos e de expectativas. Malhotra (2001) aponta outras vantagens como a sua facilidade de aplicação e de entendimento do participante sobre como respondê-la. Mas tais vantagens não impedem, contudo, a existência de problemas metodológicos na sua utilização, especialmente quando se trata de avaliação da qualidade de serviços de saúde.

A literatura discute também dificuldades relativas a aspectos metodológicos que devem ser considerados quando são realizadas pesquisas sobre atitudes de pacientes ou beneficiários de serviços médicos. Os problemas metodológicos mais comuns incluem amostras não representativas, escalas não validadas e intervalos de medida inconsistentes (Spicer, 2002). Todavia as dificuldades técnicas encontradas para a avaliação da qualidade de serviços médicos não devem desencorajar a busca de critérios cada vez mais consistentes e confiáveis. Mesmo tendo problemas metodológicos, a técnica de escala de atitudes mostra-se útil, prática e amplamente utilizada na identificação de percepções ou nível de satisfação das pessoas com relação a serviços de saúde.

Como exemplos brasileiros de escalas validadas, podemos citar a QPC, que visa medir a qualidade percebida pelo paciente de serviços médicos de consultório (Urdan, 2001) e a SATIS-BR, destinada à avaliação da satisfação dos usuários de serviços de saúde mental, elaborada pela Organização Mundial de Saúde e adaptada para o Brasil por Bandeira, Pitta e Mercier (2000). Além destes pode-se incluir o estudo realizado por Milan e Trez (2005) destinado a identificar os atributos dos serviços que compõem a avaliação da satisfação dos associados de um plano de saúde, bem como a propor um modelo de estrutura de constructos para pesquisa de satisfação em sistemas de medicina de grupo. Milan e Trez desenvolveram uma escala intervalar de sete pontos e os fatores, ou constructos, extraídos a partir da análise fatorial e regressão múltipla, envolveram o atendimento aos clientes, a estrutura (instalações, equipamentos), os médicos (confiança e segurança), o preço do plano (relação custo/benefício), a 
comunicação da administração do plano com os seus usuários e a conveniência (localização dos hospitais/ consultórios e coberturas do plano).

\section{Método}

Os dados deste estudo foram colhidos e analisados a partir das respostas de 280 trabalhadores de uma indústria de calçados localizada no Rio Grande do Sul, na região do pólo calçadista, escolhidos através de amostragem aleatória estratificada.

A empresa contava com, aproximadamente, 800 trabalhadores, sendo $58 \%$ do sexo masculino e $42 \%$ feminino. Da composição da amostra, $62 \%$ eram do sexo masculino e $38 \%$ do sexo feminino. Para assegurar a proporcionalidade da amostra por área da empresa buscou-se um equilíbrio sendo, então, 36\% representantes da área de produção, $34 \%$ da área administrativa e 30\% da área comercial. Todos os trabalhadores eram participantes de um plano de saúde gerenciado pela própria empresa, através da sua diretoria de recursos humanos com a supervisão de uma comissão constituída por trabalhadores e contando com o suporte de uma consultoria externa especializada. A participação no plano se dava pela livre adesão de cada um, podendo incluir ou não os seus dependentes. Como o plano era gerido pela própria empresa, os seus custos, naturalmente, eram mais reduzidos, sendo que o valor médio mensal da contribuição de cada empregado, incluindo seus dependentes, era da ordem de U\$12,00. A parte restante, necessária para cobrir as despesas totais do plano, era arcada pela empresa. O plano oferecia cobertura integral para todas as especialidades médicas, internações hospitalares, exames sem restrições e atendimento odontológico.

Os dados referentes às percepções relacionadas aos serviços do plano de saúde foram colhidos através de uma escala de Likert de 05 (cinco) pontos, construída especialmente para este fim, observando-se os passos propostos por Pasquali (1999). A versão original da escala apresentava 34 itens, agrupados em 8 fatores, relacionados mais adiante. Parte dos itens foi formulada na direção positiva e parte na negativa. $\mathrm{Na}$ apuração dos resultados, os itens negativos tinham, naturalmente, sua pontuação convertida. Os itens foram elaborados a partir da literatura específica (Donabedian, A. 1980, 1990; Lam, S.S.K, 1997; Urdan, A. T. 2001) e de relatos, através de comunicação pessoal, de experiências de usuários de planos de saúde relacionadas a aspectos específicos envolvendo a qualidade dos serviços. Os itens originais foram validados semanticamente, através da leitura de cada um deles para um grupo de doze trabalhadores representantes da população na qual seria, posteriormente, aplicada a escala. Após a leitura de cada item, cada trabalhador expressou, com suas próprias palavras, o significado por ele atribuído ao item em questão. Todo item que apresentava mais de um significado ou provocava interpretações diversas era reformulado ou excluído. Após sua validação semântica, os itens foram apresentados no instrumento numa ordem aleatória, a fim de minimizar possíveis efeitos de contaminação de um item para outro.

O instrumento foi entregue para cada participante em envelope acompanhado de uma carta do responsável pelo departamento de recursos humanos da empresa, enfatizando a importância da pesquisa. O índice de retorno da escala preenchida foi de $98 \%$. Nas instruções para responder aos itens do instrumento foi assegurada a confidencialidade das respostas individuais. Os participantes indicavam seu grau de concordância ou discordância com relação a cada item, assinalando um dos pontos na escala de 1 (discordo totalmente) a 5 (concordo totalmente).

$\mathrm{O}$ instrumento respondido foi encaminhado pelo participante no dia seguinte, em envelope lacrado e sem qualquer identificação pessoal, para o setor de recursos humanos da empresa.

\section{Resultados}

\subsection{Consistência Interna}

Com base no coeficiente Alpha de Cronbach, a escala total apresentou inicialmente o índice de 0,8974. Contudo, a extração da carga fatorial após a rotação dos fatores, sugeriu baixa convergência de dois itens. Com a eliminação daqueles itens, a consistência interna sofreu leve redução ( $\alpha=8879$ ), mas mantendo-se, mesmo assim, consideravelmente alta. 


\subsection{Analise Fatorial}

Para determinar a adequação da utilização do método de análise fatorial, extraiu-se o índice KMO (Kaiser-Meyer-Okin) que foi de 0,869, sugerindo a viabilidade do método para análise dos dados coletados, conforme Kaiser (1974). Com propósito semelhante, efetuou-se o teste de Bartlett e obteve-se o valor de 2544,386 confirmando a pertinência do modelo fatorial (Norusis, 1994).

Tabela 1 - Resultados da análise fatorial inicial e após a rotação

\begin{tabular}{|c|c|c|c|c|c|c|}
\hline \multirow[b]{2}{*}{ Fator } & \multicolumn{3}{|c|}{ Extração inicial } & \multicolumn{3}{|c|}{ Após a rotação dos fatores } \\
\hline & Autovalor & $\begin{array}{c}\% \\
\text { variância } \\
\end{array}$ & $\begin{array}{c}\% \\
\text { cumulativo }\end{array}$ & Autovalor & $\begin{array}{c}\% \\
\text { variância } \\
\end{array}$ & $\begin{array}{c}\% \% \\
\text { cumulativo }\end{array}$ \\
\hline 1 & 8,739 & 25,701 & 25,701 & 4,079 & 11,998 & 11,998 \\
\hline 2 & 1,957 & 5,755 & 31,457 & 2,227 & 6,550 & 18,548 \\
\hline 3 & 1,760 & 5,178 & 36,634 & 2,221 & 6,533 & 25,081 \\
\hline 4 & 1,443 & 4,244 & 40,878 & 2,052 & 6,036 & 31,117 \\
\hline 5 & 1,347 & 3,962 & 44,840 & 1,934 & 5,690 & 36,807 \\
\hline 6 & 1,304 & 3,835 & 48,675 & 1,929 & 5,674 & 42,481 \\
\hline 7 & 1,223 & 3,597 & 52,272 & 1,770 & 5,207 & 47,688 \\
\hline 8 & 1,145 & 3,368 & 55,640 & 1,762 & 5,184 & 52,872 \\
\hline 9 & 1,088 & 3,199 & 58,839 & 1,609 & 4,731 & 57,603 \\
\hline 10 & 1,038 & 3,052 & 61,892 & 1,458 & 4,288 & 61,892 \\
\hline
\end{tabular}

Fonte: Os autores

A média das comunalidades (quantidade de variância que um item ou variável compartilha com todos os demais itens) foi de 0,618. Todos os itens apresentaram comunalidade final superior a 0,40.

Partindo da concepção inicial do instrumento, os itens foram construídos e agrupados em 8 fatores, como seguem: (1) Competência: capacidade profissional dos prestadores de serviços e precisão dos exames e diagnósticos; (2) Satisfação: atendimento das necessidades dos usuários; (3) Segurança: valorização, confiança e garantias proporcionadas pelos serviços; (4) Atendimento: tratamento humano dispensado aos usuários, pelos profissionais do plano; (5) Agilidade: rapidez com que se realiza a prestação dos serviços; (6) Valorização: contribuição do plano para fortalecimento do senso de valor pessoal; (7) Administração: custo arcado pelo usuário para participação no plano e adequação das normas que o regem e (8) Motivação: estímulo para o trabalho proporcionado pelo plano.

A extração da carga fatorial inicial sugeriu, no entanto, a possibilidade de 10 fatores, tendo o de menor carga um peso de 1,038 da variância total explicada. Acima de 10 fatores os autovalores caem para menos de 1,0 desaconselhando, portanto, a aceitação de um número de fatores maior do que este. Ademais, com 10 fatores já foi possível explicar mais de $60 \%$ da variância total existente no conjunto das variáveis presentes na concepção inicial do instrumento.

Mesmo constatando-se uma distribuição relativamente equilibrada das variâncias explicadas pelos fatores, notou-se na análise inicial que a distância entre o primeiro fator $(25,7)$ e o segundo $(5,7)$ era mais acentuada. Através da rotação ortogonal Varimax foi possível encontrar uma melhor distribuição dos percentuais das variâncias explicadas pelos fatores, como pode ser verificada na tabela abaixo, que apresenta a situação após a extração inicial dos fatores e após a rotação dos mesmos.

\subsubsection{Composição dos fatores}

Os itens agrupados, a partir das suas cargas fatoriais observadas após a rotação dos fatores, aplicando o método Varimax com normalização de Kaiser, ficaram com a composição identificada na Tabela 2. Nesta tabela pode observar, também, que os itens 3 e 31, por apresentarem carga fatorial menor do que 0,4, não ficaram ligados a qualquer fator.

Os itens 1, 6 e 9 aparecem em dois fatores ao mesmo tempo. Nestes casos a carga fatorial deve ser o critério de decisão preferencial. Contudo, a natureza do item também deve ser considerada, especialmente quando seu conteúdo se ajusta melhor num determinado grupo do que em outro. Este parece ser o caso do item 9 que, mesmo tendo carga fatorial mais elevada no fator $2(0,469$ para 0,444$)$, seu conteúdo mostrase mais compatível com o fator 3 . 
Tabela 2 - Matriz dos fatores rotacionados

\begin{tabular}{|c|c|c|c|c|c|c|c|c|c|c|c|c|}
\hline \multirow[b]{2}{*}{ Item } & \multirow[b]{2}{*}{ Média D } & \multirow[b]{2}{*}{$p$} & \multicolumn{10}{|c|}{ Fator } \\
\hline & & & 1 & 2 & 3 & 4 & 5 & 6 & 7 & 8 & 9 & 10 \\
\hline 23 & 4,40 & $\phi, 94$ & 0,714 & & & & & & & & & \\
\hline 10 & 4,56 & $\emptyset, 85$ & 0,687 & & & & & & & & & \\
\hline 19 & 4,00 & 7,21 & 0,670 & & & & & & & & & \\
\hline 32 & 4,26 & 7,13 & 0,624 & & & & & & & & & \\
\hline 30 & 3,99 & 7,30 & 0,620 & & & & & & & & & \\
\hline 11 & 4,01 & ,25 & 0,593 & & & & & & & & & \\
\hline 25 & 4,61 & $\phi, 74$ & 0,489 & & & & & & & & & \\
\hline 20 & 3,55 & 7,23 & & 0,734 & & & & & & & & \\
\hline 4 & 4,51 & 7,01 & & 0,559 & & & & & & & & \\
\hline 6 & 3,94 & 7,35 & & 0,523 & & 0,454 & & & & & & \\
\hline 9 & 3,93 & 4,27 & & 0,469 & 0,444 & & & & & & & \\
\hline 31 & 4,42 & $\oint, 91$ & & & & & & & & & & \\
\hline 8 & 4,39 & $\Phi, 95$ & & & 0,714 & & & & & & & \\
\hline 24 & 3,95 & 7,05 & & & 0,658 & & & & & & & \\
\hline 16 & 4,20 & 7,15 & & & 0,629 & & & & & & & \\
\hline 29 & 4,63 & $\oint, 91$ & & & & 0,763 & & & & & & \\
\hline 34 & 4,49 & $\oint, 88$ & & & & 0,639 & & & & & & \\
\hline 15 & 4,28 & $\emptyset, 93$ & & & & & 0,673 & & & & & \\
\hline 14 & 4,02 & 7,23 & & & & & 0,646 & & & & & \\
\hline 28 & 4,23 & $\emptyset, 95$ & & & & & 0,536 & & & & & \\
\hline 26 & 3,76 & 7,34 & & & & & & 0,759 & & & & \\
\hline 21 & 4,18 & 7,28 & & & & & & 0,605 & & & & \\
\hline 27 & 3,16 &, 50 & & & & & & 0,558 & & & & \\
\hline 13 & 4,42 & 7,18 & & & & & & & 0,756 & & & \\
\hline 7 & 4,05 & 40 & & & & & & & 0,689 & & & \\
\hline 2 & 2,96 & 7,47 & & & & & & & 0,506 & & & \\
\hline 5 & 4,16 & 1,12 & & & & & & & & 0,737 & & \\
\hline 33 & 4,61 & $\oint, 81$ & & & & & & & & 0,656 & & \\
\hline 22 & 4,58 & $\phi, 89$ & & & & & & & & 0,503 & 0,420 & \\
\hline 12 & 4,61 & $\Phi, 80$ & & & & & & & & & 0,594 & \\
\hline 17 & 4,61 & $\oint, 97$ & & & & & & & & & 0,589 & \\
\hline 3 & 4,66 & $\phi, 81$ & & & & & & & & & & \\
\hline 18 & 4,30 & 7,03 & & & & & & & & & & 0,767 \\
\hline 1 & 4,31 & 1,12 & 0,417 & & & & & & & & & 0,588 \\
\hline
\end{tabular}

Fonte: Ss autores

A tabela $\beta$ descreve os fatores com seus respectivos itens agrupados segundo os critérios expostos acima

Tabela $\beta$ - Fatores e itens

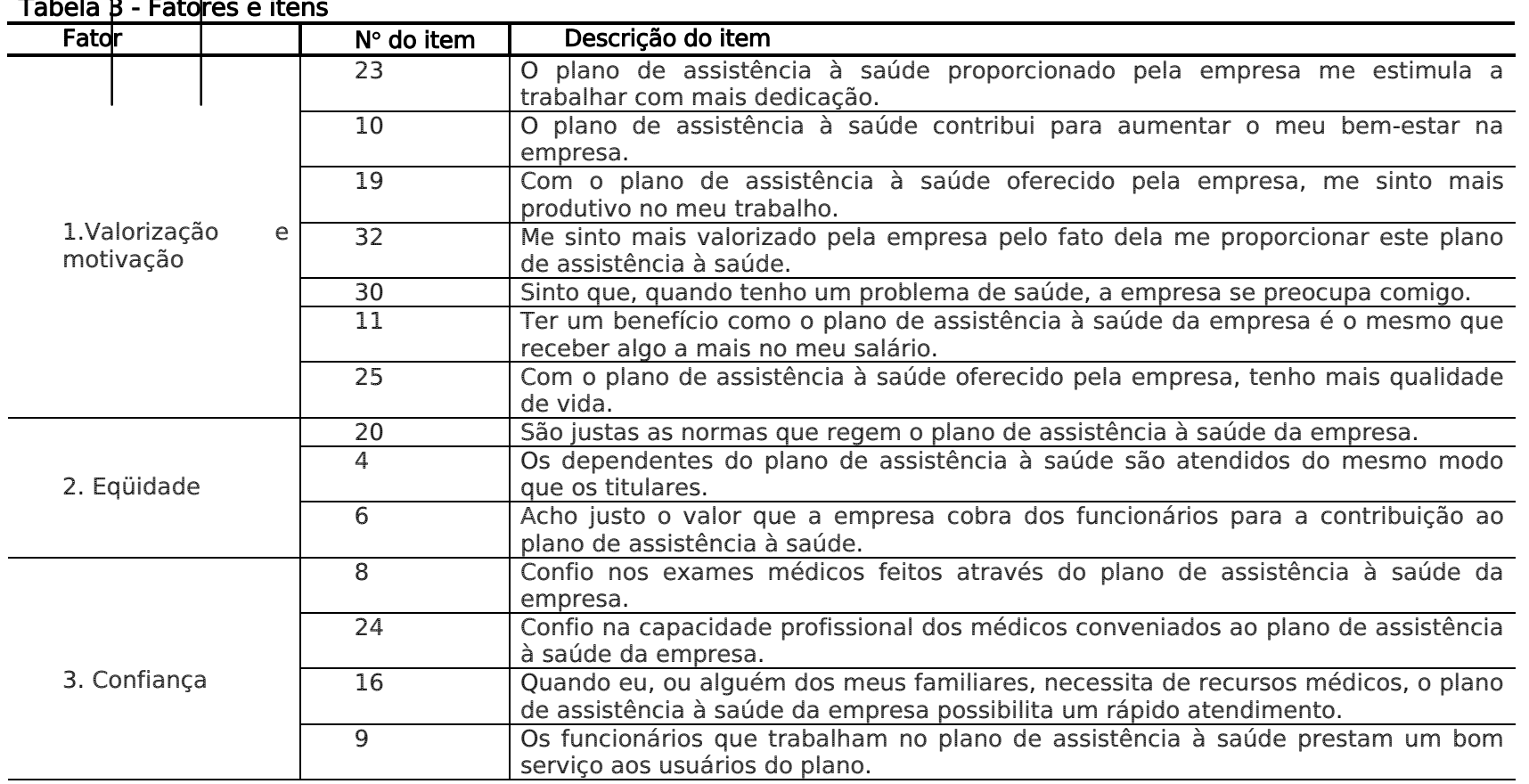


Cont. Tabela 3 - Fatores e itens

\begin{tabular}{|c|c|c|}
\hline \multirow[t]{2}{*}{ 4. Custo } & 29 & $\begin{array}{l}\text { Seria mais barato para mim não participar da contribuição para o plano de } \\
\text { assistência à saúde da empresa e arcar eu mesmo com as despesas de tratamento } \\
\text { médico. }\end{array}$ \\
\hline & 34 & $\begin{array}{l}\text { O plano de assistência à saúde oferecido pela empresa me proporciona economia } \\
\text { de dinheiro. }\end{array}$ \\
\hline \multirow{3}{*}{ 5. Atendimento } & 15 & $\begin{array}{l}\text { O atendimento humano dado pelas clínicas conveniadas satisfaz as minhas } \\
\text { necessidades. }\end{array}$ \\
\hline & 14 & $\begin{array}{l}\text { Participando do plano de assistência à saúde da empresa significa que serei melhor } \\
\text { atendido pelos postos conveniados. }\end{array}$ \\
\hline & 28 & $\begin{array}{l}\text { A qualidade do atendimento humano dos hospitais conveniados atende minhas } \\
\text { expectativas. }\end{array}$ \\
\hline \multirow{3}{*}{$\begin{array}{l}\text { 6. Consideração e } \\
\text { fidelização }\end{array}$} & 26 & Sou mais considerado como ser humano por ter este plano de assistência à saúde. \\
\hline & 21 & $\begin{array}{l}\text { Ter o plano de assistência à saúde da empresa contribui para que eu falte menos } \\
\text { ao trabalho. }\end{array}$ \\
\hline & 27 & $\begin{array}{l}\text { Um dos motivos que me faz permanecer na empresa é o seu plano de assistência à } \\
\text { saúde. }\end{array}$ \\
\hline \multirow{3}{*}{$\begin{array}{l}\text { 7. Agilidade e } \\
\text { valorização } \\
\text { familiar }\end{array}$} & 13 & Meus familiares dão pouco valor ao plano de assistência à saúde da empresa. \\
\hline & 7 & $\begin{array}{l}\text { Quando usei o plano de assistência à saúde fui mal atendido pelos médicos } \\
\text { conveniados. }\end{array}$ \\
\hline & 2 & O processo de encaminhamento médico aos especialistas é lento demais. \\
\hline \multirow{3}{*}{$\begin{array}{l}\text { 8. Fortalecimento } \\
\text { da empresa }\end{array}$} & 5 & $\begin{array}{l}\text { O fato de ter o plano de assistência à saúde da empresa faz com que eu ajude a } \\
\text { empresa a crescer cada vez mais. }\end{array}$ \\
\hline & 33 & $\begin{array}{l}\text { Ao oferecer um plano de assistência à saúde aos seus funcionários entende-se que } \\
\text { a empresa está, de alguma forma, contribuindo para o desenvolvimento do país. }\end{array}$ \\
\hline & 22 & $\begin{array}{l}\text { A empresa que oferece um plano de assistência à saúde aos seus funcionários tem } \\
\text { mais vantagens para competir no mercado. }\end{array}$ \\
\hline \multirow[b]{2}{*}{ 9. Segurança } & 12 & $\begin{array}{l}\text { Com o plano de assistência à saúde me sinto mais seguro com relação ao dia de } \\
\text { amanhã. }\end{array}$ \\
\hline & 17 & $\begin{array}{l}\text { Para mim não faz grande diferença ter ou não um plano de assistência à saúde } \\
\text { como este oferecido pela empresa. }\end{array}$ \\
\hline \multirow[t]{2}{*}{ 10. Proteção } & 18 & $\begin{array}{l}\text { A cobertura do plano em termos de tipos de tratamentos oferecidos garante o } \\
\text { atendimento das minhas necessidades principais. }\end{array}$ \\
\hline & 1 & $\begin{array}{l}\text { O plano de assistência à saúde me faz lembrar que devo cuidar melhor de mim } \\
\text { mesmo. }\end{array}$ \\
\hline
\end{tabular}

Fonte: Os autores

\subsection{Validade convergente}

Para proceder a validação convergente utilizou-se como variável dependente a média global dos itens e como variáveis independentes os índices para cada um dos dez fatores, calculados pela média dos escores de suas respectivas variáveis. Através da análise de regressão linear múltipla obteve-se um coeficiente de determinação ajustado significativo $(99,5)$ indicando que grande parte da variância dos resultados globais é explicada pelos índices dos 10 fatores que compõem a escala. A tabela 4 mostra a força dos índices dos fatores da escala como preditores da percepção global dos trabalhadores. Observa-se que o maior coeficiente $(0,317)$ refere-se ao fator 1 (Valorização e motivação), distanciando-se consideravelmente dos demais fatores.

Tabela 4 - Análise de regressão dos resultados globais sobre os fatores

\begin{tabular}{lccc}
\hline \multicolumn{1}{c}{ Fatores } & $\begin{array}{c}\text { Coeficientes não } \\
\text { padronizados }\end{array}$ & $\begin{array}{c}\text { Coeficientes } \\
\text { padronizados }\end{array}$ & $\mathrm{t}$ \\
\hline (Constante) & 0,024 & & $1,011^{*}$ \\
\hline 1. Valorização e motivação & 0,218 & 0,317 & $45,518^{* *}$ \\
\hline 2. Eqüidaade & 0,095 & 0,162 & $29,389^{* *}$ \\
\hline 3. Confiança & 0,127 & 0,187 & $34,378^{* *}$ \\
\hline 4. Custo & 0,062 & 0,088 & $16,716^{* *}$ \\
\hline 5. Atendimento humano & 0,094 & 0,140 & $28,061^{* *}$ \\
\hline 6. Fidelização & 0,088 & 0,179 & $31,393^{* *}$ \\
\hline 7. Agilidade & 0,093 & 0,164 & $33,786^{* *}$ \\
\hline 8. Fortalecimento da empresa & 0,091 & 0,116 & $22,224^{* *}$ \\
\hline 9. Segurança & 0,064 & 0,081 & $15,572^{* *}$ \\
\hline 10. Proteção & 0,068 & 0,111 & $22,547^{* *}$ \\
\hline
\end{tabular}

$* p>0,05, * * p<0,001$. Fonte: Os autores

\section{Discussões dos resultados e conclusão}

A proposta de um instrumento destinado especificamente a avaliar a qualidade do plano de saúde da empresa e de um modelo que Ihe dá suporte, suscita questões originadas tanto dos pressupostos básicos 
do modelo ora proposto, quanto dos dados empíricos colhidos neste estudo inicial de verificação de validade.

A literatura oferece alguns argumentos favoráveis aos planos de saúde, especialmente porque eles contribuem para atrair e reter empregados mais qualificados (Buchmueller \& Valetta, 1999; Kinard, 2001) e tornando-os mais satisfeitos no seu trabalho. Se estes pressupostos possuem apoio empírico, ainda é questão aberta, porquanto a maior parte dos autores parece basear suas afirmações muito mais na lógica do senso comum do que na evidência trazida pela análise sistemática de dados extraídos sob condições controladas.

É razoável reconhecer que os gestores das empresas mantenham expectativas de retorno deste investimento sob a forma de atitudes positivas dos beneficiários do plano de saúde, com relação ao seu trabalho e a própria empresa. Identificar estas atitudes pode tornar-se uma importante fonte de subsídios para a formulação das estratégias da empresa, relacionadas aos seus recursos humanos. 0 retorno esperado conforme sugerido pelo modelo aqui proposto se constitui na qualidade agregada do plano de saúde que é o equivalente atitudinal da qualidade percebida. Diante disto, seria lícito esperar que, quanto maior a qualidade percebida, maior a qualidade agregada. Esta é uma hipótese a ser testada num próximo estudo.

Outra linha de questionamento é inspirada pelos resultados obtidos através da análise fatorial, particularmente quanto à composição dos fatores extraídos após a rotação. Percebe-se que, na maior parte das vezes, a composição sugerida para os diferentes fatores reuniu itens cujo conteúdo era de natureza semelhante. Todavia, isto nem sempre ocorreu, especialmente no fator "Agilidade", o qual reuniu itens de natureza completamente diversa. Coincidência ou não, tais itens estavam formulados na direção negativa, o que poderia estar determinando a convergência para um mesmo fator. Por outro lado, há indícios de dificuldades para responder de forma apropriada a esses itens. Giacomoni (2002) verificou que crianças e pré-adolescentes encontraram dificuldades para interpretação de itens formulados na direção negativa. Embora os participantes deste estudo pertencessem a faixas etárias da vida adulta, muitos deles não possuíam além do ensino fundamental, o que poderia também dificultar a correta interpretação destes itens. Esta é, contudo, uma questão a ser também investigada em futuros estudos.

Cabe ressalvar que a escala composta através deste estudo piloto constitui-se em uma versão preliminar, sujeita a aperfeiçoamentos provocados por novas análises. Como sinalizadores das qualidades positivas desta escala e do seu potencial técnico, destacam-se o seu alto grau de consistência interna e a sua validade convergente, as quais confirmam a importância de seguir investigando as suas propriedades psicométricas.

Adicionalmente deve-se considerar que este estudo foi realizado numa população de trabalhadores de uma empresa de calçados, participante de um plano de saúde coletivo empresarial auto-administrado. Esta característica pode significar que o instrumento em questão só será aplicável em empresas do mesmo ramo e com planos de saúde da mesma natureza. Estas particularidades também sugerem a necessidade de novos estudos.

Finalmente, é importante salientar que, no levantamento da literatura sobre o impacto do plano de saúde sobre as atitudes dos trabalhadores não foram encontrados instrumentos, em forma de escalas, para mensuração destas atitudes. Por esta razão este estudo pode inspirar, também, pesquisas sobre o tema.

\section{Referências}

Bandeira, M., Pitta, A. M. F. \& Mercier, C. (2000). Escala de avaliação da satisfação dos usuários em serviços de saúde mental: SATIS-BR. Jornal Brasileiro de Psiquiatria, 49 (8), pp 293-300.

Bowers, M., Swan, J. E., \& Hoekler, W. F. (1994). What attributes determine quality and satisfaction with health care delivery? Health Care Manager Review, 19 (4), pp. 49-55. PMid:7896552

Buchmueller, T.C., \& Valletta, R.G. (1999). The effect of health insurance on married female labor supply. The Journal of Human Resources, 34 (1), pp. 42-70. doi: 10.2307/146302

Corrêa, S., Piola, S., \& Arilha, M. (1998). Cairo em ação: estudo de caso Brasil. New York: Population Reference Bureau (mimeo). 
Cronin, J., \&Taylor, S. (1992). Measuring service quality: A reexamination and extension. Journal of Marketing, 56 (3), pp. 55-68. doi: 10.2307/1252296

Donabedian, A. (1980). La calidad de la atención médica: definición y métodos de evaluación. Mexico: La Prensa Médica Mexicana.

Donabedian, A. (1990). The seven pillars of quality. Archives of Pathology and Laboratory Medicine, Northfield, v. 114 (11), pp. 115-1118.

Gerschman, S., Veiga, L., Guimarães, C., Ugá, M. A. D., Portela, M. C., Vasconcellos, M. M., Barbosa, P. R., Lima, S. M. L. L. (2007). Estudo de satisfação dos beneficiários de planos de saúde de hospitais filantrópicos. Ciência \& saúde coletiva, 12 (2).

Giacomoni, C. H. (2002). Bem-estar subjetivo infantil: conceito de felicidade e construção de instrumentos para avaliação. UFRGS: Tese de Doutorado, Pós-Graduação em Psicologia do Desenvolvimento, Universidade Federal do Rio Grande do Sul, Brasil.

Gronroos, C. (1995). Marketing, gerenciamento e serviços: a competição por serviços na hora da verdade. Rio de Janeiro: Campus.

Kaiser, H. F. (1974). An index of factorial simplicity. Psychometrika, 39 (1), pp. 31-36. doi: 10.1007/BF02291575

Kilsztajn, S., Câmara, M. B., \& Carmo, M.S.N. (2001). Gasto privado com saúde por classes de renda. XXIV General Population Conference/IUSSP.

Kinard, J. (200l). An examination of employer-provided benefits in the health care industry The Health Care Manager, 18 (4), pp. 55-62.

Lam, S. S. K. (1997). Servqual: A tool for measuring patients' opinions of hospital service quality in Hong Kong. Total Quality Management, 8 (4), Aug., pp. 145-152. doi: 10.1080/0954412979587. PMCid:2860396

Malhotra, N. (2001). Pesquisa de marketing: uma orientação aplicada. 3a ed., PA: Bookman.

MERIC, H. J. (1994). The effect of scale form choice on psychometric properties of patient satisfaction measurement. Health Marketing Quarterly, 11 (3-4), pp. 27-39. PMid:10137016

Milan, G. S., \& Trez, G. (2005). Pesquisa de satisfação: um modelo para planos de saúde. RAE-eletrônica, 4 (2).

Norusis, M. J. (1994). SPSS professional statistic 6,1. Chicago: SPSS.

Pasquali, L. (1999). Escalas psicométricas. In Pasquali, L. (org). Instrumentos Psicológicos: manual prático de elaboração. Brasília: LabPAM; IBAPP.

Righi, A. W., Schmidt, A. S., \& Venturini, J. C. (2010). Qualidade em serviços públicos de saúde: uma avaliação da estratégia saúde da família. Revista Produção Online 10 (3), pp. 649-669.

Spicer, J. (2002). How to measure patient satisfaction. QualityProgress, 35 (2), pp. 97-98.

Sternberg, R. J. (2008). Psicologia cognitiva. Porto Alegre: Artmed.

Urdan, A. T. (2001). A qualidade de serviços médicos na perspectiva do cliente. Revista de Administração de Empresas, 41 (4), pp. 44-55. 